Artigo

\title{
Programa de prácticas para la formación inicial de profesores de matemáticas en la Universidad Surcolombiana
}

\author{
Practice program for the initial training of teachers of mathematics at the Universidad \\ Surcolombiana
}
Programa de práticas de formação inicial de professores de matemática da Universidade Sulcolombiana

\author{
Martha Cecilia Mosquera Urrutia ${ }^{1}$ \\ [0000-0003-0091-1034]
}

\begin{abstract}
Resumo
São apresentados os resultados do processo de pesquisa interinstitucional: "Desenho e gestão de um programa de estágio para a formação inicial de professores nas faculdades de educação de duas universidades latino-americanas" cujo objetivo geral era desenhar e administrar um programa de estágios que promovesse a flexibilidade a cooperação curricular, académica, a promoção da investigação e da formação integral dos alunos e os seus objetivos específicos na caracterização das práticas, conceção e gestão do programa. A primeira parte do artigo dá conta da gênese do projeto e da conformação da comunidade de aprendizagem e prática, a segunda, dos referenciais teóricos e metodológicos para caracterizar as práticas, a terceira, da utilização de um modelo teórico para caracterizá-los, o quarto da proposta de programa e o quinto, da gestão do programa de licenciatura em matemática de uma universidade participante. Entre os resultados estão: a formação de comunidades de aprendizagem e prática a nível disciplinar, interdisciplinar e interinstitucional, o posicionamento das práticas como eixo fundamental da formação inicial de professores e a consolidação do congresso internacional de experiências na formação de professores como cenário disseminar o conhecimento que emerge da prática e dos processos de pesquisa; promover processos de reflexão e sistematização a partir das práticas pedagógicas e investigativas; redimensionar o caráter investigativo nelas imerso e promover o interinstitucionalismo nos processos de formação inicial e continuada de professores.
\end{abstract}

Palavras-chave: Racionalidades. Práticas de ensino. Comunidades de aprendizagem e prática.

\begin{abstract}
Results of the inter-institutional research process are presented: "Design and management of an internship program for the initial training of teachers in the faculties of education of two Latin American universities" whose general objective was to design and manage an internship program that promotes flexibility curricular, academic cooperation, the promotion of research and comprehensive training in students and their specific objectives in characterizing practices, designing and managing the program. The first part of the article gives an account of the genesis of the project and the conformation of the learning and practice community, the second, of the theoretical and methodological references to characterize the practices, the third, of the use of a theoretical model to characterize them, the fourth of the program proposal and the fifth, of the management of the program designed for the degree in mathematics of a participating university. Among the results are:

${ }^{1}$ martha.mosquera@usco.edu.co, Doutorado em Didática da Matemática, Coordenador do Centro de Pesquisa de Excelência em Qualidade da Educação CIECE e Professor do Curso de Licenciatura em Matemática, Universidade Surcolombiana, Neiva/Huila/Colombia.
\end{abstract}


the formation of learning and practice communities at a disciplinary, interdisciplinary and interinstitutional level, the positioning of practices as a fundamental axis of initial teacher training and the consolidation of the international congress of experiences in teacher training as a scenario to disseminate the knowledge that emerges from the practice and research processes; promote reflection and systematization processes from pedagogical and investigative practices; resizing the investigative character immersed in them and promoting inter-institutionalism in the processes of initial and ongoing teacher training.

Keywords: Rationalities. Teaching practices. Communities of learning and practice.

\section{Resumen}

Se presentan resultados del proceso de investigación interinstitucional: "Diseño y gestión de un programa de prácticas para la formación inicial de profesores en las facultades de educación de dos universidades latinoamericanas" cuyo objetivo general consistió en diseñar y gestionar un programa de prácticas que promueva la flexibilidad curricular, la cooperación académica, el fomento de la investigación y la formación integral en los estudiantes y sus objetivos específicos en caracterizar las practicas, diseñar y gestionar el programa. La primera parte del artículo da cuenta de la génesis del proyecto y la conformación de la comunidad de aprendizaje y práctica, la segunda, de los referentes teóricos y metodológicos para caracterizar las prácticas, la tercera, del uso de un modelo teórico para caracterizarlas, la cuarta de la propuesta del programa y la quinta, de la gestión del programa diseñado para la licenciatura en matemáticas de una universidad participante. Entre los resultados se cuentan: la conformación de comunidades de aprendizaje y práctica a nivel disciplinar, interdisciplinar e interinstitucional, el posicionamiento de las prácticas como eje fundamental de la formación inicial del profesorado y la consolidación del congreso internacional de experiencias en formación de profesores como escenario para divulgar el conocimiento que emerge de los procesos de práctica e investigación; fomentar procesos de reflexión y sistematización desde las prácticas pedagógicas e investigativas; redimensionar el carácter investigativo inmerso en ellas y promover la interinstitucionalidad en los procesos de formación inicial y continua de profesores.

Palabras claves: Racionalidades. Prácticas de enseñanza. Comunidades de aprendizaje y práctica

\section{Introducción}

El proyecto de investigación interinstitucional del que da cuenta el presente artículo, se concretó después de varias sesiones de trabajo, en las cuales se fue configurando una comunidad de aprendizaje y práctica con el objetivo de discutir a cerca de las tensiones que se hacían evidentes por las normativas ministeriales en relación con los espacios de práctica y la presencia de las mismas en los planes de estudio de los programas de formación inicial de profesores, dicha comunidad está conformada por los decanos de las facultades de educación de las tres universidades que conformaban en 2014 el Capítulo Suroriente de la Asociación Colombiana de Facultades de Educación, en adelante ASCOFADE, los coordinadores de práctica de cada una de las facultades y de los programas académicos, y un docente investigador o el coordinador de investigaciones de cada uno de dichos programas.

El proceso de investigación ha permitido una reflexión continua sobre las prácticas, el uso de marcos teóricos y metodológicos para caracterizar las prácticas, el fortalecimiento de los programas de formación de profesores, así como también la readacción de varios artículos, algunos de ellos posicionados en revistas indexadas, en revistas institucionales y en capítulos de libros resultado de investigación. 


\section{Antecedentes del proyecto:}

Las primeras reuniones que se relacionan, se dieron entre profesores de los programas de las licenciaturas en matemáticas y matemáticas y física, de las universidades Surcolombiana y Amazonia, con el fin de discutir sobre problemas referentes a las prácticas, a los recientes resultados de investigación y a la normatividad frente a las licenciaturas.

Posteriormente, se realizaron varias reuniones promovidas por los decanos de las facultades de educación del Capítulo Sur Oriente de ASCOFADE, a las que se vincularon algunos coordinadores de práctica, asesores y cooperadores, con el fin de estudiar las propuestas del Ministerio de Educación frente a las licenciaturas. Se realizó un primer encuentro de experiencias de práctica en Ibagué (Universidad del Tolima), en el cual se socializaron experiencias significativas y reflexiones sobre los retos para los programas en relación con los procesos de autoevaluación con fines de acreditación o registro calificado y se discutieron problemas propios de la formación de los profesores por parte de estudiantes y docentes de las tres universidades, de ahí sugió la idea de organizar el primer encuentro de experiencias significativas en educación.

Este encuentro, fue organizado en 2015 y se definió como un espacio para conocernos, reconocernos y generar aprendizajes a partir de la investigación y de nuestras propias experiencias. En ese orden de ideas, se consolidaron tanto la definición, como el objetivo que hoy en día se mantienen: "Promover un escenario académico que permita socializar el conocimiento que emerge de los procesos de práctica desarrollados por los profesores en formación, como posibilidad de fortalecer la formación de los profesores como ciudadanos con capacidad de actuar profesionalmente en los contextos escolares, con sensibilidad social e intercultural y habilidades para integrar saberes en la búsqueda de soluciones a problemas de los contextos, preservando un equilibrio con la propia cultura, el sistema educativo y la sociedad que se quiere construir".

Durante el encuentro se socializaron cuarenta y cinco experiencias: 23 en el área de matemáticas y física, 9 en el área de ciencias naturales: física, química y biología, 4 en literatura y lengua castellana, 2 en educación artística y cultural, 1 en pedagogía infantil, 3 de establecimientos educativos de básica y media, 3 de maestría en educación y una conferencia nacional.

Desde esa época se empezaron a registrar experiencias de práctica que utilizan marcos teóricos de referencia para analizar problemas de enseñanza-aprendizaje y generar conocimiento a partir de la investigación sobre las prácticas; destaco algunos que fueron utilizados en varias experiencias:

La Teoría del Análisis Didáctico, que en síntesis "se refiere a la conceptualización de las actividades que el profesor de matemáticas debería realizar para diseñar, llevar a la práctica y evaluar unidades didácticas" (GÓMEZ, 2006, pág. 34), dicho análisis es específico de un contenido matemático concreto y le permite al profesor en primer lugar, identificar los múltiples significados de un concepto y tomar desiciones sobre aquellos que serán objeto de instrucción. El análisis didáctico se divide en cuatro análisis (contenido, cognitivo, instrucción y actuación) los cuales le permiten al profesor abordar estos dos aspectos. Algunas experiencias particularmente de la Universidad de la Amazonia muestran el uso del marco en el contexto de las áreas de matemáticas y física.

El marco del Conocimiento Profesional del Profesor, que distigue dos tipologías de conocimiento: el Conocimiento del Contenido (CC) y el Conocimiento Didáctico (CD). El 
Conocimiento del Contenido incluye: el Conocimiento Común del Contenido (CCC), el Conocimiento Propedéutico (CP) y el Conocimiento Especializado del Contenido (CEC) y el Conocimiento Didáctico del Contenido (CDC) que incluye también tres componentes: el Conocimiento del Contenido y de los Alumnos (CCA), Conocimiento del Contenido y de la Enseñanza (CCE) y el Conocimiento del Contenido y del Currículo (CCC), alrededor de este marco se han tejido variados estudios en los últimos años y en el marco del encuentro fue utilizado principalmente por profesores de Ciencias Naturales y Matemáticas y entre las refrencias comunes a Shulman y a Ball. (SHULMAN, 1986; BALL, 1991; AMORTEGUI \& CORREA, 2012; AMÓRTEGUI, 2011).

La Investigación como Estrategia Pedagógica del Deparatamento Administrativo de Ciencia y Tecnología en adelante COLCIENCIAS (hoy Ministerio de Ciencia Tecnología e Innovación MINCIENCIAS): en algunas experiencias de práctica basadas en procesos de investigación que muestran como implementar en el aula iniciativas puntuales del Programa ONDAS de COLCIENCIAS, en especial se hizo alusión a la formación de Maestros investigadores a través del uso de la Caja de Herramientas para Maestros y Maestras ONDAS (COLCIENCIAS, 2007) y los cinco cuadernillos: El lugar de maestros y maestras en ONDAS (Manjarrés \& Mejía, 2007), La Pregunta Como Punto de Partida y Estrategia Metodológica (Manjarrés \& Mejía, 2007), La Investigación Como Estrategia Pedagógica IEP (MANJARRÉS \& MEJÍA, 2007), Producción de Saber y Conocimiento en los Maestros y Maestras ONDAS (MANJARRÉS \& MEJÍA, 2007), y Las Comunidades de Aprendizaje: Prácticas, Conocimiento, Saber y Transformación. Apoyadas en las Nuevas Tecnologías de la Información y la Comunicación (MANJARRÉS \& MEJÍA, 2011).

Otros tópicos fueron: el Estudio de Clases (ISODA \& OLFOS, 2009), Aprendizaje Basado en Problemas (BARROWS, 1996), las comunidades de aprendizaje (MEN, 2016); Diseño, gestión y evaluación de tareas matemáticas (PTA, 2015); estudios sobre las creencias de estudiantes y profesores en relación con la enseñanza y el aprendizaje de conceptos principalmente de álgebra, proyectos pedagógicos y atención a poblaciones con discapacidad auditiva (CALDERÓN \& LEÓN, 2016).

Además de las experiencias, se ofrecieron tres talleres y se tocaron temas transversales como el uso de TICS, la formación de maestros investigadores, las creencias de los profesores y de los futuros profesores, el uso de materiales didácticos y educativos, las prácticas de acompañamiento y las prácticas extramuros.

Para cerrar este encuentro se desarrolló el primer panel de expertos: las prácticas pedagógicas y su impacto en la formación de profesores, esto es importante porque propició la delimitación de tres líneas de trabajo una a cargo de cada universidad participante, así la Universidad Surcolombiana asumió el papel de las prácticas en la formación inicial de profesores, la Universidad del Tolima las prácticas pedagógicas y su articulación con la investigación en la formación inicial de profesores y la Universidad de la Amazonia las prácticas pedagógicas como una posibilidad para hacer visible la internacionalización del currículo, (Fig. 1) esto nos permitió revisar aspectos normativos, teóricos y resultados de investigación que posteriormente aportan al marco legal del Programa diseñado. (SOCIAL \& MOSQUERA-URRUTIA, 2020, p.50) 
Figura 1 - Líneas de trabajo para el panel.

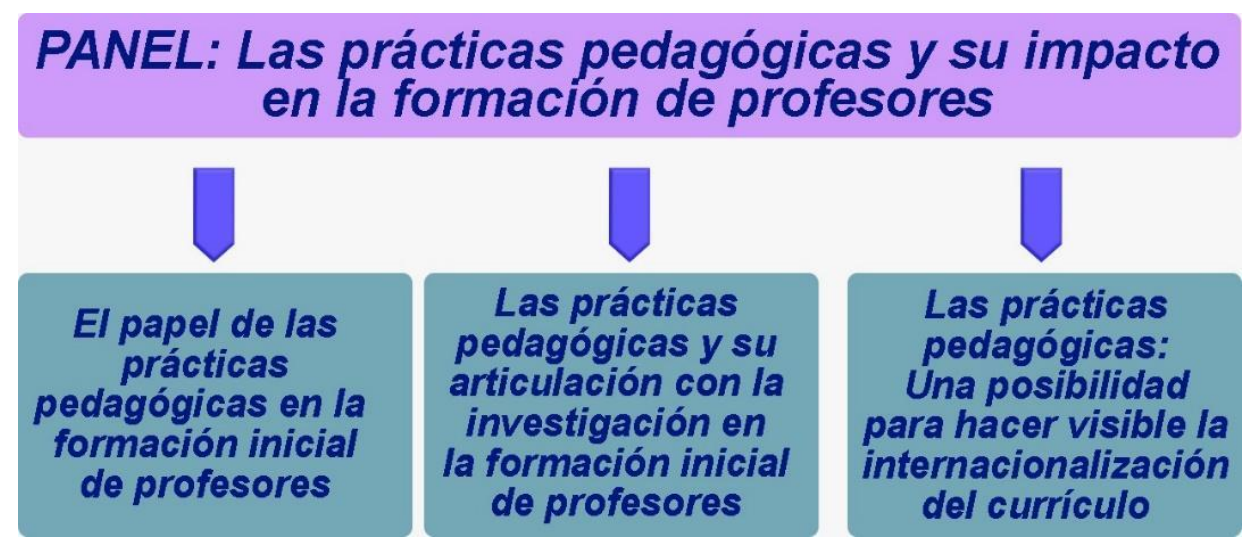

Fuente: Elaboración propia

\section{Referente teórico y metodológico del programa de prácticas}

Una vez conformada la comunidad de aprendizaje e identificada la problemática, se dio inicio al proyecto de investigación interistitucional: "Diseño y gestión de un programa de prácticas para la formación inicial de professores, en las facultades de educación de dos universidades latinoamericanas" cuyo objetivo general consistió en diseñar y gestionar un programa de prácticas pedagógicas que promueva la flexibilidad curricular, la cooperación académica, el fomento de la investigación y la formación integral de los estudiantes. Y como objetivos específicos:

a. Caracterizar las prácticas pedagógicas de los programas académicos de la Facultad de Educación de las universidades participantes.

b. Diseñar un programa de práctica pedagógica que promueva la flexibilidad curricular, la cooperación académica, el fomento de la investigación y la formación integral en los estudiantes para los programas académicos de la Facultad de Educación de la Universidad de la Amazonia.

c. Gestionar el programa de prácticas

De entre las posibles fuentes que permiten caracterizar las prácticas de formación de los profesores, se tuvieron en cuenta:

- Los discursos se encuentran en las explicaciones, descripciones, propuestas que se hacen sobre el ejercicio profesional, estos pueden ser orales o escritos, personales $\mathrm{o}$ institucionales y subyacen tanto en el Proyecto Educativo universitario, el Proyecto Educativo de cada licenciatura, los microdiseños curriculares, planes de área, planes de práctica, reflexiones, etc.

- Las actuaciones se refieren a las actividades que realizan en el ejercicio de la profesión

- Las racionalidades son las razones que sustentan los discursos sobre la actividad profesional y la actuación como docentes en los contextos escolares

En la investigación se asume la racionalidad como aquello "dotado de razón, es decir, dotado de sentido y no derivado del azar", (LATORRE; 2006; p.23), es posible indagar por los discursos y por las actuaciones de profesores en formación en los diferentes programas académicos, toda vez que, siguiendo los planteamientos de Boudon (2003), particularmente 
sobre las actuaciones de los individuos, estas se sustentan en las siguientes premisas: a) Son de naturaleza diversa, b) se producen en contextos y c) en estos contextos asumen roles particulares y establecen relaciones de saber y de poder. (Acuerdos logrados en el grupo de investigación)

Lo anterior "implica reconocer que cada individuo cuando realiza una acción o cree en alguna cosa, lo hace porque dicha acción o dicha creencia tiene sentido para él y él significa, adhiere o actúa en función de este sentido, porque tiene "buenas razones" para ello" (LATORRE; 2006; p. 23), o en otros términos, las concepciones y creencias que tengan los profesores están determinando sus discursos y sus actuaciones. Para el caso particular, de la primera fase de la investigación, "caracterización de las prácticas pedagógicas", el interés se centra en los discursos explícitos en los documentos que institucionalizan las prácticas pedagógicas: El proyecto curricular, los microdiseños curriculares y las producciones de los estudiantes.

Se asume como modelo de análisis el propuesto en 2005 por Marisol Latorre en su investigación "Aportes para el análisis de las racionalidades presentes en las prácticas pedagógicas" el cual se articula a partir de cuatro componentes: Este modelo se articula a partir de cuatro componentes 1) "buenas razones" y racionalidades, 2) naturaleza teóricopráctica de la acción educativa, 3) teorías y saberes asociados a la acción educativa y 4) características de las prácticas pedagógicas (LATORRE, 2004, P.6). Para el análisis de las racionalidades Latorre toma los tres postulados básicos de la Teoría de la Elección Racional (BOUDON 2003), mencionado por (LATORRE, 2004, P.7)

Una de las razones por la que se decidió elegir este modelo es que los criterios y categorías definidas permiten caracterizar y analizar las racionalidades presentes en las prácticas pedagógicas, tanto del campo de la formación inicial como del campo del ejercicio docente en la escuela, lo que hace posible avanzar en el desarrollo de un análisis comparativo. (P. 7)

En el modelo, los componentes o dimensiones se pueden expresar a través de la existencia de dos constructos al interior de todo hecho educativo: una teoría de la educación y una práctica educativa. En otras palabras, cada vez que se observa, estudia o analiza un hecho educativo es posible reconocer que en él están presentes teoría y práctica a la vez (P. 9) y que interactúan. (Fig. 2)

Figura 2 - Teoría y práctica niveles y relaciones

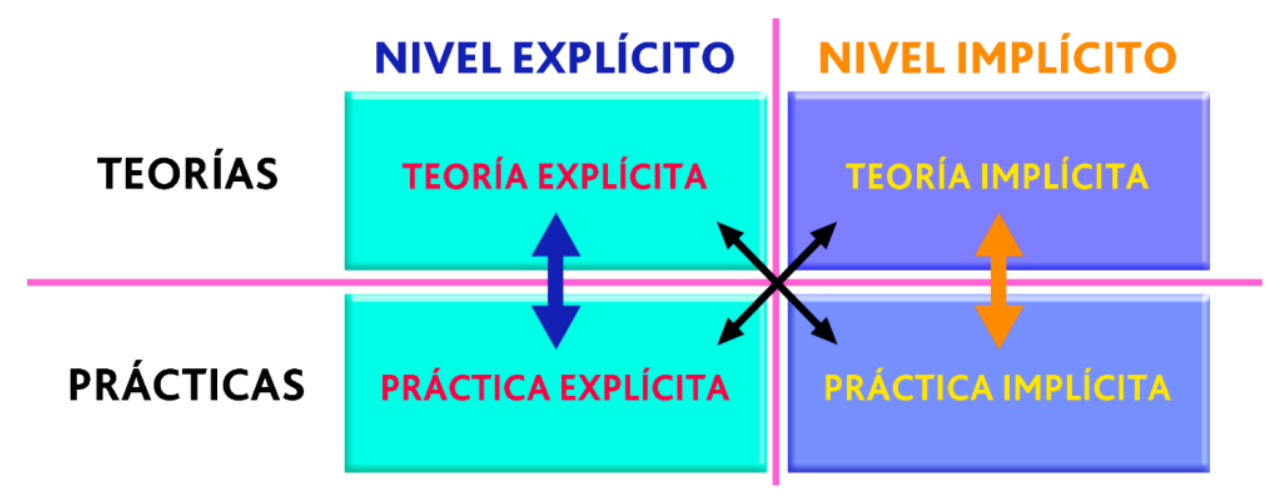

Fuente: LATORRE, 2004. P. 9 


\section{Categorías de análisis para caracterizar las prácticas pedagógicas}

Se distinguen en relación con la naturaleza del programa académico tres tipologías de saberes: saber empírico, saber práctico y saber teórico especulativo; en relación con el objeto de conocimiento: saber personal, saber local, saber general; en relación con la práctica: saber utilitario instrumental, saber normativo y orientador guiado y de acuerdo con la posibilidad de explicitación saber declarativo, procedimental y, explícitos y comunicables (Fig. 3).

Figura 3 - Categorías para caracterizar las prácticas pedagógicas en la formación inicial de profesores propuesta por Latorre (2006)

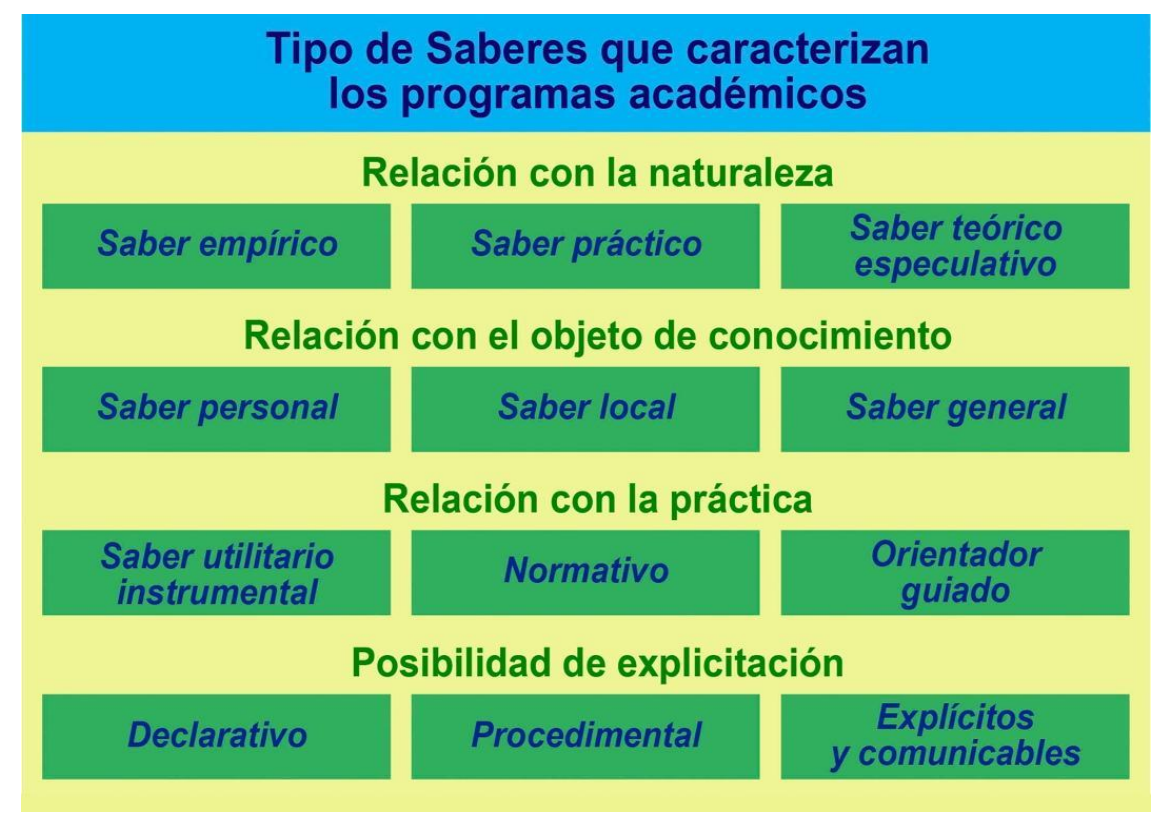

Fuente: Elaboración Propia.

De acuerdo con los discursos y las actuaciones de los profesores en formación es posible indagar aspectos de las prácticas pedagógicas como: ¿Concepciones explícitas en el Programa Académico? ¿ Propósitos que sustentan las PP? ¿Finalidad del saber en relación con los propósitos de las PP? ¿Tipo de saberes epistemológicos sustentan las PP? ¿Saberes pedagógicos y didácticos sustentan las PP? ¿Contenidos estructuran los desarrollos curriculares de las PP? ¿Nivel de estructuración de estos contenidos? ¿Diálogo entre los saberes teóricos y los saberes prácticos? ¿Estructura metodológica de las PP? ¿Estrategias de objetivación, formalización y explicitación del aprendizaje? ¿Relación entre docencia e investigación desde las PP? ¿Características de la evaluación del aprendizaje desde las PP?

Responder a estas preguntas permite describir las prácticas pedagógicas. De acuerdo a la tipificación que hace Mialaret (1995), de las prácticas pedagógicas, la caracterización de los saberes explícitos e implícitos en los discursos y actuaciones de los profesores en formación, permiten tipificarlas como experienciales, reproductivas y transformativas. El siguiente cuadro caracteriza cada tipo de práctica en relación con los saberes que las sustentan (Fig. 4). Las fuentes de información son: Proyecto educativo de programa PEP, Microdiseños de las asignaturas y las multitareas. 
Figura 4:Tipo de práctica en relación con los saberes que las sustentan (Mialaret 1995)

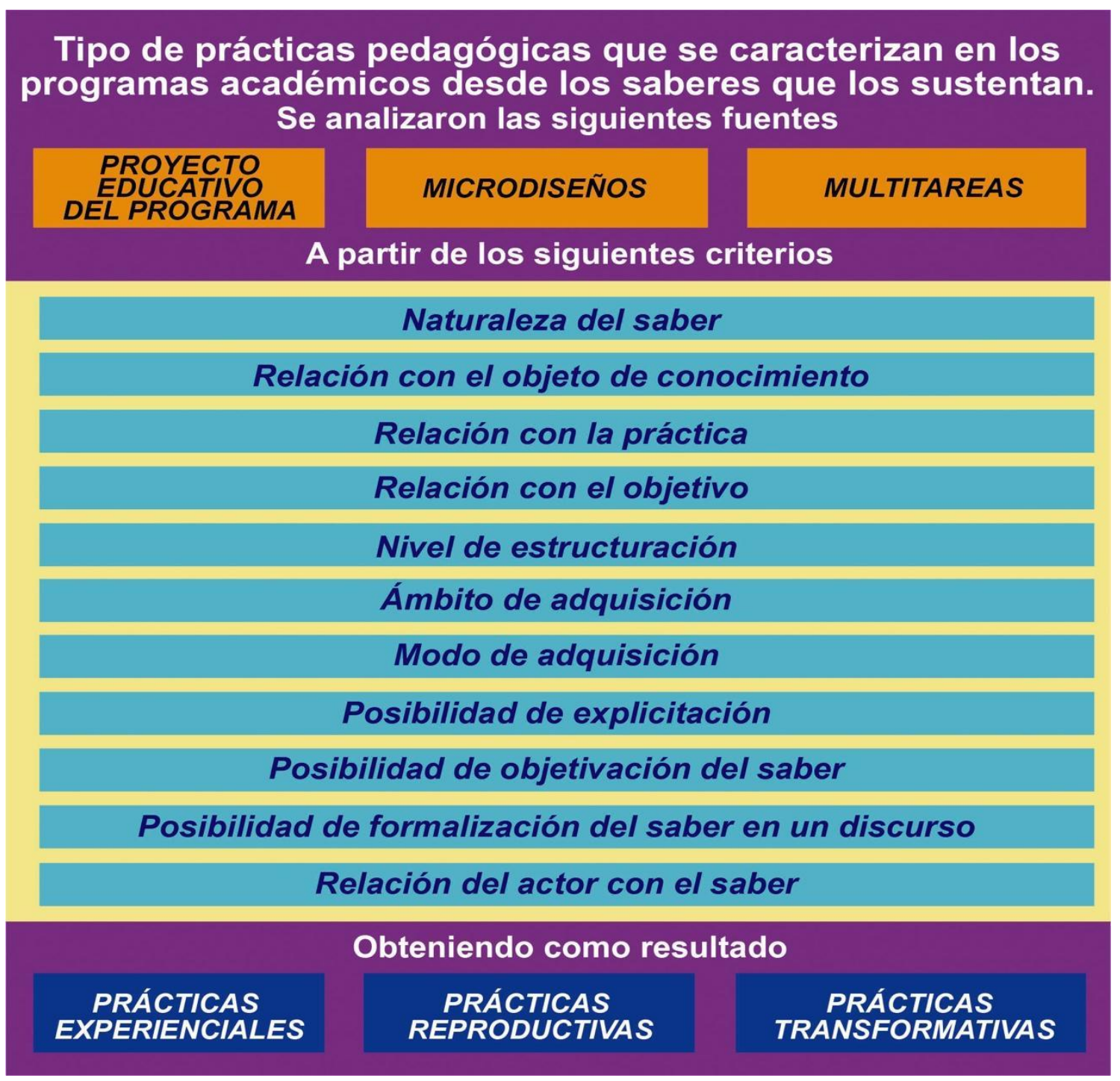

Fuente: Elaboración propia

En igual forma se caracterizan las prácticas de acuerdo con las actuaciones y desde las mismas fuentes que en el caso anterior. (Fig. 5) 
Figura 5 - Tipo de práctica en relación con los saberes que las sustentan (MIALARET, 1995)

$\begin{gathered}\text { Tipo de prácticas pedagógicas que se caracterizan en los } \\ \text { programas académicos desde las actuaciones. } \\ \text { Se analizaron las siguientes fuentes }\end{gathered}$
$\begin{gathered}\text { PROYECTO } \\ \text { EDUCATIVO } \\ \text { MEL PROGRAMA }\end{gathered}$
A partir de los siguientes criterios
Principal propósito de la acción
Fundamento principal de la acción
Nivel de estructuración de la acción
Criterio de planificación de la acción
Respuesta frente a situaciones problemáticas
Vínculos con prácticas de análisis
Vínculos con prácticas investigativas
Naturaleza del aprendizaje posible en el profesor/a
Criterios de evaluación de la acción práctica
Lógica operante en la práctica
Obteniendo como resultado
PRÁCTICAS
REPRODUCTIVAS

Fuente: Elaboración propia

\section{Referente metodológico para caracterizar las prácticas pedagógicas}

Tratando de identificar referentes metodológicos para describir los discursos y las actuaciones de los profesores en formación para caracterizar sus prácticas, logramos identificar algunas investigaciones que comparten el objeto de estudio de esta investigación:

En la investigación ¿Qué actividades de enseñanza utilizan los profesores en formación inicial y los profesores en ejercicio cuando planifican unidades didácticas en ciencias?, desarrollada por Pro Bueno, Saura y Sánchez (2000), se plantean algunos elementos interesantes sobre las prácticas de los profesores, los autores señalan que la planificación de unidades didácticas -cuando se desvincula de una mera exigencia administrativa-, puede facilitar información relevante sobre respecto a lo que "realmente piensa" el profesor en relación con diversos aspectos de su práctica profesional. De alguna forma esta postura de los autores se acerca a los planteamientos de Latorre, respecto a las buenas razones que los profesores en formación otorgan a sus discursos y sus actuaciones lo que caracteriza sus prácticas. (Fuente: Documento de trabajo) 
Los investigadores solicitaron a los profesores en formación diseñar unidades didácticas del área de ciencias, que posteriormente gestionarían y evaluarían en el aula, donde realizan sus prácticas, el diseño debía contemplar cinco tareas en las que habían incidido a partir del desarrollo de un curso de formación. Los autores centraron su interés en dar respuesta a los siguientes interrogantes ¿Qué tipo de actividades utilizan los profesores en formación inicial cuando planifican una unidad didáctica de ciencias? ¿Qué contenidos procedimentales están implícitos en las actividades de enseñanza que plantean los futuros profesores? ¿Existe alguna relación entre los tipos de actividades y los contenidos procedimentales implicados en las planificaciones de los profesores?

Los investigadores realizaron categorizaciones de los tipos de actividades que realizaron los profesores en formación en el diseño de las unidades didácticas, centraron su atención en analizar cuatro aspectos: a) tipo de actividades, b) duración de las actividades, c) contenidos conceptuales implicados y d) contenidos procedimentales. Los investigadores agruparon las actividades en función del protagonismo de los implicados en el proceso, a continuación, se registran las actividades agrupadas por cada tipo de protagonismo.

Protagonismo centrado en el profesor: orientación, exposición del profesor, utilización de medios audiovisuales, ejercicios realizados por el profesor en la pizarra, uso de mapas conceptuales y resumen del tema.

Protagonismo compartido: experiencias de cátedra, trabajo del profesor con el gran grupo/puesta en común, programa guía y clases de dudas sobre el tema.

Protagonismo centrado en el alumno: explicitación de ideas, lectura de un texto, actividades individuales de los alumnos (casi siempre de papel y lápiz), trabajo en pequeños grupos, trabajo de laboratorio, revisión de ideas y tareas para casa.

A continuación se presenta el listado de actividades que fueron reportadas en el marco de dicha investigación (Tabla. 1)

Tabla 1 - Listado de actividades que registran los profesores en formación en sus planeaciones (PRO BUENO, SAURA \& SÁNCHEZ, 2000)

\begin{tabular}{|c|l|}
\hline COD. & Descripción de la actividad \\
\hline ORI & Orientación al desarrollo de un tema \\
\hline EXP & Exposición del profesor \\
\hline MAV & Uso de medios audiovisuales \\
\hline MAP & Elaboración de un mapa conceptual por el profesor \\
\hline RES & Resumen del tema realizado por el profesor \\
\hline CAT & Realización de experiencias de cátedra \\
\hline TGG & Trabajo del profesor con el gran grupo/puesta en común \\
\hline GUI & Utilización de un programa-guía \\
\hline DUD & Clases de dudas de los alumnos sobre el tema \\
\hline IDE & Explicitación de ideas \\
\hline LEC & Lectura de un texto por los alumnos \\
\hline IND & Realización de actividades individuales por los alumnos \\
\hline LAB & Realización de actividades de laboratorio \\
\hline TPG & Trabajo en pequeño grupo (no actividad practica) \\
\hline REV & Revisión de ideas \\
\hline OAC & Otras actividades \\
\hline TAR & Tareas para casa \\
\hline
\end{tabular}

Fuente: elaboración propia 
Con los referentes planteados propusimos una estructura metodológica adaptada a los intereses del proyecto de investigación. Latorre sugiere caracterizar las prácticas pedagógicas a partir de los discursos personales, institucionales y las actuaciones, la caracterización se desarrolla desde la teoría de las buenas razones que propone la autora, en la tabla siguiente se muestra la forma como se asume el proceso de caracterización para los discursos personales. La tabla incorpora como categoría de análisis la tipología de prácticas sugeridas por Mialaret, citadas por Latorre, se pretende en el proceso de caracterización identificar el tipo de prácticas que está presente en los discursos y las actuaciones de los profesores en formación inicial.

La tabla Nro. 2 registra como unidad de análisis los discursos personales, las categorías conceptuales que se proponen son los componentes del currículo (contenidos, objetivos, metodología y evaluación) que sustentan los procesos de planificación de los profesores. Los indicadores de análisis corresponden a los tipos de prácticas que emergen de los discursos de los profesores en formación asociados a cada categoría, los descriptores se formulan desde los aportes de Latorre en torno a las "buenas razones" que surgen de los argumentos implícitos en la multitarea que se asume como fuente de análisis.

Tabla 2 - Estructura metodológica para caracterizar la práctica pedagógica desde los discursos personales sustentada en la teoría de las buenas razones (Latorre 2006)

\begin{tabular}{|c|c|c|c|c|c|}
\hline $\begin{array}{l}\text { Unidad } \\
\text { de }\end{array}$ & $\begin{array}{l}\text { Categoría de } \\
\text { análisis }\end{array}$ & \multicolumn{2}{|c|}{ Indicadores de análisis } & $\begin{array}{l}\text { Descriptores de } \\
\text { análisis }\end{array}$ & $\begin{array}{c}\text { Fuente de } \\
\text { análisis }\end{array}$ \\
\hline \multirow[b]{5}{*}{$\begin{array}{l}\text { Discurso } \\
\text { personal }\end{array}$} & \multirow{3}{*}{ Contenidos } & $\begin{array}{l}\text { (PE) Practica } \\
\text { experiencial/saber } \\
\text { empírico }\end{array}$ & & $\begin{array}{l}\text { (EI) Creencias e } \\
\text { ideas previas }\end{array}$ & \\
\hline & & $\begin{array}{l}(\mathrm{PR}) \quad \text { Practica } \\
\text { reproductiva/saber } \\
\text { practico }\end{array}$ & & $\begin{array}{l}\text { (II) Intereses } \\
\text { institucionales }\end{array}$ & \\
\hline & & $\begin{array}{l}\text { (PT) Practica } \\
\text { transformativa/saber } \\
\text { teórico-especulativo }\end{array}$ & & $\begin{array}{l}\text { (IPD) Intereses } \\
\text { pedagógicos, } \\
\text { didácticos } \\
\text { culturales }\end{array}$ & \\
\hline & \multirow{2}{*}{ Objetivos } & $\begin{array}{l}\text { (PE) Practica } \\
\text { experiencial/saber } \\
\text { empírico }\end{array}$ & & $\begin{array}{l}\text { (RS) Replicar un } \\
\text { saber } \\
\text { individual/replicar } \\
\text { un saber validado } \\
\text { por una } \\
\text { comunidad } \\
\text { científica } \\
\end{array}$ & \\
\hline & & $\begin{array}{l}\text { (PR) } \quad \text { Practica } \\
\text { reproductiva/saber } \\
\text { practico }\end{array}$ & $\begin{array}{l}\text { Argumentos } \\
\text { asociados a: }\end{array}$ & $\begin{array}{l}\text { (CIP) Conocer e } \\
\text { informar } \\
\text { procedimientos, } \\
\text { reglas y } \\
\text { normas/predecir } \\
\text { resultados } \\
\text { favorables del } \\
\text { curso }\end{array}$ & Multitarea \\
\hline
\end{tabular}




\begin{tabular}{|c|c|c|}
\hline & $\begin{array}{l}\text { (PT) Practica } \\
\text { transformativa/saber } \\
\text { teórico-especulativo }\end{array}$ & $\begin{array}{l}\text { (DCC) Describir } \\
\text { una } \\
\text { realidad/Conocer } \\
\text { para comprender } \\
\text { la realidad desde } \\
\text { diferentes } \\
\text { perspectivas }\end{array}$ \\
\hline \multirow{3}{*}{ Metodología } & $\begin{array}{l}\text { (PE) Practica } \\
\text { experiencial/saber } \\
\text { empírico }\end{array}$ & $\begin{array}{l}\text { (DAE) Diseño de } \\
\text { actividades } \\
\text { sustentadas en la } \\
\text { experiencia } \\
\text { personal }\end{array}$ \\
\hline & $\begin{array}{l}\text { (PR) Practica } \\
\text { reproductiva/saber } \\
\text { practico }\end{array}$ & $\begin{array}{l}\text { (DAC) Diseño de } \\
\text { actividades } \\
\text { derivadas de la } \\
\text { lógica del } \\
\text { contenido }\end{array}$ \\
\hline & $\begin{array}{l}\text { PT) Practica } \\
\text { transformativa/saber } \\
\text { teórico-especulativo }\end{array}$ & $\begin{array}{l}\text { (DAP) Diseño de } \\
\text { actividades } \\
\text { sustentadas en la } \\
\text { pertinencia } \\
\text { necesidad } \\
\text { contexto }\end{array}$ \\
\hline \multirow{3}{*}{ Evaluación } & $\begin{array}{l}\text { (PE) Practica } \\
\text { experiencial/saber } \\
\text { empírico }\end{array}$ & $\begin{array}{l}\text { (Al) Verificar nivel } \\
\text { de aprendizajes } \\
\text { individuales } \\
\text { logrados }\end{array}$ \\
\hline & $\begin{array}{l}\text { (PR) Practica } \\
\text { reproductiva/saber } \\
\text { practico }\end{array}$ & $\begin{array}{l}\text { (AR) Identificar } \\
\text { nivel de alcance } \\
\text { de resultados } \\
\text { previstos por el } \\
\text { profesor }\end{array}$ \\
\hline & $\begin{array}{l}\text { (PT) Practica } \\
\text { transformativa/saber } \\
\text { teórico-especulativo }\end{array}$ & $\begin{array}{l}\text { (APE) Criterios de } \\
\text { evaluación } \\
\text { definidos a partir } \\
\text { de las } \\
\text { perspectivas de } \\
\text { los diferentes } \\
\text { participantes }\end{array}$ \\
\hline
\end{tabular}

Fuente: elaboración propia

\section{Resultados}

En el programa de licenciatura en matemáticas de la Universidad Surcolombiana, se hicieron tres aplicaciones de la multitarea, la primera en el curso de Didáctica de la matemática I (2017 - 02) 13 registros, la siguiente en la práctica docente I $(2018-1) 21$ registros, y en la práctica docente II (2018 - 02) 11 registros, para un total de 45 registros.

Para el caso de estudio solamente se tomaron como unidad de análisis el discurso personal y las actuaciones de los profesores, como fuente de análisis la multitarea y como categorías de análisis: contenidos, objetivos, metodología y evaluación en los discursos personales y: socializador, instructor, formador para las actuaciones. 


\section{Análisis de la información}

En la tabla Nro. 3 se puede ver que la mayor tendencia está hacia las prácticas reproductivas con un total de 28 registros (49\%), seguidas por las prácticas experienciales con 11 registros (38\%) y solamente 6 registros (13\%) de prácticas transformativas.

Tabla 3 - Resultados de aplicación de la multitarea

\begin{tabular}{|c|c|c|c|c|c|}
\hline \multirow[b]{2}{*}{ Unidad de análisis } & \multirow[b]{2}{*}{$\begin{array}{l}\text { Fuente de } \\
\text { análisis }\end{array}$} & \multirow[b]{2}{*}{$\begin{array}{c}\text { Categoría de } \\
\text { análisis }\end{array}$} & \multicolumn{3}{|c|}{ Tipología de prácticas } \\
\hline & & & $\begin{array}{l}\text { PE Práctica } \\
\text { experiencial }\end{array}$ & $\begin{array}{l}\text { PR Práctica } \\
\text { reproductiv } \\
\text { a }\end{array}$ & $\begin{array}{c}\text { PT Práctica } \\
\text { transformati } \\
\text { va }\end{array}$ \\
\hline \multirow{4}{*}{ Discurso personal } & \multirow{7}{*}{ Multitarea } & Contenidos & & & \\
\hline & & Objetivos & 11 & 28 & 6 \\
\hline & & Metodología & $38 \%$ & $49 \%$ & $13 \%$ \\
\hline & & Evaluación & & & \\
\hline \multirow{3}{*}{ Actuaciones } & & Socializador & & & \\
\hline & & Instructor & & & \\
\hline & & Formador & & & \\
\hline
\end{tabular}

Fuente: elaboración propia

En cuanto a las buenas razones dadas por los profesores en formación para argumentar los discursos personales se evidencia que:

- Se presenta una secuencia de contenidos declarativos, que corresponde a un "sector" de la matemática. Los profesores que eligieron enseñar a "calcular el área de un trapecio", presentan el contenido en el nivel de las figuras geométricas, pero no dan cuenta de las razones que justifican la elección de este contenido. La cuestión "calcular el área de un trapecio" no va más allá de la descripción de la figura geométrica o de alguna de sus cualidades. Siguiendo a Gascón (2003) nos encontramos ante "una cuestión encerrada en sí misma y por tanto muerta"

- En relación con los objetivos se busca principalmente que los estudiantes aprendan el contenido y los procedimientos correspondientes "calcular o ejercitar". Pero por ejemplo la cuestión "medir" que pudiera dar sentido a la presencia de este objeto en el currículo no es considerada en las multitareas.

- Las metodologías que se proponen pretenden que los estudiantes resuelvan las cuestiones propuestas con base en las explicaciones que ha ofrecido el profesor, por ejemplo deducir una fórmula para calcular el área o descomponer y recomponer el trapecio. $Y$

- Los criterios y estrategias de evaluación están centrados en la reproducción de los contenidos y procedimientos enseñados por el profesor.

Después de hacer el análisis de estas multitareas y de la caracterización de sus prácticas de acuerdo a las racionalidades presentes en ellas decidimos que nos encotrábamos frente al fenómeno didáctico que Gascón denomina: autismo temático, que se presenta cuando en la institución escolar, documentos, libros de texto o programas de curso, hay un encierro en los contenidos a nivel matemático, que no permite evidenciar las razones de ser de su estudio en la escuela, lo más "peligroso" de este fenómeno, es que ocasiona una división 
entre lo disciplinar y lo pedagógico entendido como "el ámbito de actuación del profesor". (GASCÓN, 2003)

\section{Programa de prácticas para la formación inicial de profesores de matemáticas en la Universidad Surcolombiana}

De acuerdo con los resultados del proyecto de investigación, el objetivo general del componente de enseñanza y aprendizaje consiste en lograr que los profesores en formación inicial desarrollen capacidades, conocimiento y habilidades para migrar del actual modelo centrado en la enseñanza de contenidos declarativos, a la comprensión y aplicación de un modelo centrado en el desarrollo del aprendizaje autónomo y construyan conocimiento sobre: los fenómenos de enseñanza y aprendizaje, los fenómenos que le dan sentido a un campo del saber, la construcción del conocimiento y los valores relacionados con el desarrollo de la cultura matemática en la escuela y la sociedad y desarrollen capacidades para planear, gestionar y evaluar unidades didácticas.

Las reflexiones de los cursos se abordan desde varios ángulos: ¿cómo se ha enseñado tradicionalmente? ¿cómo se enseña en la actualidad? ¿cómo podría ser enseñado un contenido? ¿cómo se aprende? ¿qué enseñar? y ¿para qué enseñar?

Se asume que para "enseñar" a aprender matemáticas y desarrollar o reeducar el pensamiento matemático, la mediación debe realizarse en tres fases: - aprender a conocer mediante la ejecución guiada de estrategias cognitivas, - aprender a evaluar lo aprendido mediante la ejecución guiada de estrategias metacognitivas, - aprender a transferir mediante el diseño de ambientes en los que sea posible experimentar, aplicar y comunicar lo aprendido.

\section{La práctica en los espacios de enseñanza aprendizaje es problematizadora}

Su objetivo es lograr que el profesor en formación caracterice problemas de enseñanza o aprendizaje, de los objetos matemáticos ligados a los desarrollos curriculares de las instituciones educativas.

Estas prácticas no están orientadas a que los futuros profesores dirijan clases, aunque si podremos ayudar a organizar actividades o a coordinar proyectos de investigación en los cursos, teniendo en cuenta la nueva realidad, se spera que los futuros profesores puedan entablar un diálogo con los docentes de aula, a través de una encuesta con el fin de conocer:

a. Cuáles son los sentimientos que han experimentado durante este periodo de trabajo en casa

b. Logros y dificultades frente al proceso de enseñanza desde casa

c. Necesidades identificadas

Los futuros profesores tendrán un portafolios en el cual registrarán los resultados de la entrevista manera de diario de campo.

A partir de los resultados de la entrevista, generaremos la propuesta de apoyo pedagógico para los procesos de trabajo en casa, que fundamentalmente busca lograr que conocida la realidad los futuros profesores en el marco del desarrollo del curso y con el apoyo de los investigadores: docentes

a. Consulten y socialicen algunas estrategias de aprendizaje autónomo con los 
b. Identifiquen material didáctico pertinente y de fácil acceso para los estudiantes

c. Trabajen en equipo en el diseño de actividades transversales e interdisciplinarias

d. Contribuyan en el diseño de un plan de ajuste

Tabla 4 - Propuesta de programa de prácticas e inserción por semestre

\begin{tabular}{|c|c|c|c|}
\hline Denominación & Articulación & No. de Créditos & Transversalidad \\
\hline \multirow{7}{*}{ Observación } & Psicología general y evolutiva & 1 & \multirow{6}{*}{$\begin{array}{l}\text { NUCLEO DE } \\
\text { FACULTAD }\end{array}$} \\
\hline & Psicología del aprendizaje & 1 & \\
\hline & Epistemología & 1 & \\
\hline & Pedagogías & 2 & \\
\hline & Currículo & 1 & \\
\hline & $\begin{array}{c}\text { Gestión, evaluación y desarrollo } \\
\text { educativo }\end{array}$ & 2 & \\
\hline & Enseñanza y aprendizaje de la & ? & \multirow{6}{*}{ NUCLEO ESPECÍFICO } \\
\hline \multirow{2}{*}{$\begin{array}{l}\text { Práctica Educativa } \\
\text { Investigativa }\end{array}$} & aritmética y la geometría & 2 & \\
\hline & Enseñanza y aprendizaje del & 2 & \\
\hline & álgebra y la estadística & & \\
\hline Formativa & $\begin{array}{l}\text { Enseñanza y aprendizaje del } \\
\text { cálculo }\end{array}$ & 2 & \\
\hline \multirow{2}{*}{ De investigación } & $\begin{array}{c}\text { Práctica } \\
\text { Pedagógica I }\end{array}$ & 4 & \\
\hline & $\begin{array}{c}\text { Práctica } \\
\text { Pedagógica II } \\
\end{array}$ & 4 & \multirow{3}{*}{ NUCLEO ESPECÍFICO } \\
\hline Práctica Pedagógica & $\begin{array}{c}\text { Práctica } \\
\text { Pedagógica III }\end{array}$ & 4 & \\
\hline \multirow{4}{*}{ Profesional docente } & $\begin{array}{c}\text { Práctica } \\
\text { Pedagógica IV } \\
\end{array}$ & 4 & \\
\hline & Práctica profesional docente I & 6 & \multirow{3}{*}{ NUCLEO ESPECÍFICO } \\
\hline & Práctica profesional docente II & 6 & \\
\hline & Práctica profesional docente III & 6 & \\
\hline \multicolumn{4}{|c|}{48 CRÉDITOS } \\
\hline
\end{tabular}


Figura 6 - Propuesta metodológica para los cursos de enseñanza y aprendizaje

Propuesta de ruta metodológica para los espacios académicos de enseñanza aprendizaje

Fuente: elaboración propia

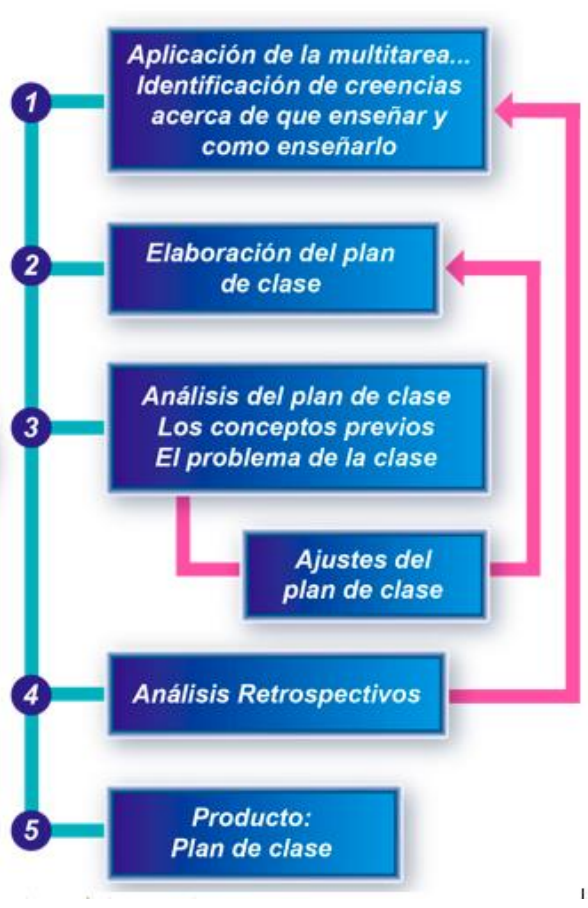

\section{Referencias}

AMÓRTEGUI, Elías; CORREA, Mónica; VALBUENA, Édgar. Aporte de las prácticas de campo a la construcción del conocimiento profesional de futuros profesores de Biología. In: Proceedings of II Congrés Internacional de Didactiques. Girona, 2010.

BALL, Deborah Loewenberg. Research on teaching mathematics: Making subject matter knowledge part of the equation. East Lansing, 1988.67p.

BOUDON, Raymond. Más allá de la teoría de la elección racional. Revista anual de sociología, v. 29, n. 1, p. 1-21, 2003.

CALDERÓN, Dora; LEÓN, Olga. Dispositivos didácticos para el desarrollo de competencia comunicativa en matemáticas. In: Calderón, Dora; León, Olga (Eds.), Elementos para una didáctica del lenguaje y las matemáticas en estudiantes sordos de niveles iniciales Investigaciones. Bogotá, Colombia: Universidad Distrital Francisco José de Caldas, p. 143-160, 2016.

GÓMEZ GUZMÁN, Pedro et al. Análisis didáctico en la formación inicial de profesores de matemáticas de secundaria. In: Investigación en educación matemática: actas del X Simposio de la Sociedad Española de Investigación en Educación Matemática. Córdoba, 2006; p. 9-25, 2006.

GÓMEZ, Pedro. Análisis Didáctico en la Formación Inicial de Profesores de Matemáticas de Secundaria. In: Bolea, María Pilar; Moreno, Mar; González, María José (Eds.), Investigación en educación matemática: actas del X Simposio de la Sociedad Española de Investigación en Educación Matemática. Huesca: Instituto de Estudios Altoaragoneses, p. 15-53, 2006. 
INAPE, Colectivo. La Sistematización de Experiencias en la Investigación Pedagógica para la Construcción de un Patrimonio de Saber Pedagógico en la Región Amazónica. Perspectivas Educativas, v. 9 , n. 1, p. 121-135, 2019.

LATORRE NAVARRO, Marisol. Aportes para el análisis de las racionalidades presentes en las prácticas pedagógicas. Estudios pedagógicos (Valdivia), n. 30, p. 75-91, 2004.

LATORRE NAVARRO, Marisol. Formación inicial de profesores: una apuesta para enfrentar los nuevos desafíos. Santiago, 2008.

LATORRE, Marisol, et al. ¿ Cuáles son las características de las prácticas pedagógicas de profesores chilenos en ejercicio? Revista digital PREAL, p. 1 - 20, 2005.

MANJARRÉS, María Elena, et al. Niños, niñas y jóvenes investigan: Lineamientos de la investigación como estrategia pedagógica. 2016.

MEJÍA, Marco Raul; CIPRIAN, Jenny; MANJARRÉS, María Elena. Manual de Apoyo a la gestión ya la construcción del Programa Ondas. Bogotá, Colombia: Prograf Itda, 2011.

MIALARET, Gaston. Réflexions personnelles sur la pratique et ses relations avec la théorie, la recherche et la formation. Cahiers de la recherche en éducation, 1995, v. 2, n. 1, p. 165-183, 1995.

NAVARRO, Marisol Latorre. Nuevas miradas, viejos problemas: Las relaciones entre formación inicial y ejercicio profesional docente. Foro Educacional, n. 10, p. 41-64, 2006.

SAURA LLAMAS, Octavio; PRO BUENO, Antonio de; SÁNCHEZ BLANCO, Gaspar. ¿̇ Qué actividades de enseñanza utilizan los profesores en formación inicial y los profesores en ejercicio cuando planifican unidades didácticas de ciencias? Revista Investigación en la Escuela, v. 40, p. 23-37, 2000.

SHULMAN, Lee S. Quienes comprenden: una concepción del conocimiento docente. Educador estadounidense, v. 10, n. 1, 1986.

SOCIAL, Proyección \& MOSQUERA-URRUTIA, Martha Cecilia 2020. 2do. CONGRESO INTERNACIONAL DE EXPERIENCIAS EN FORMACIÓN DE PROFESORES. Revista Proyección Social, v. 2, n.1, p. 45-51, 2020. Recuperado a partir de https://journalusco.edu.co/index.php/rps/article/view/2374 


\section{Anexo 1: Facsímil de multitarea}

\section{UNIVERSIDAD}

\section{-FACULTAD DE EDUCACION}

Proyecto de investigación: Diseño y gestión de un programa de prácticas para la formación inicial de Profesores en las facultades de educación de las Universidades de la Amazonia y Surcolombiana

Fecha:

Nombre:

Programa académico:

Estimado estudiante: con el propósito caracterizar las prácticas pedagógicas de los profesores en formación inicial de la facultad, agradecemos su colaboración en desarrollar las actividades propuestas a continuación.

Seleccione un contenido de su campo de formación que le gustaría abordar en el aula.

1. Elabore un mapa conceptual que muestre las temáticas, y los diferentes aspectos que considere debería abordar en el aula con sus estudiantes. ¿Qué enseñar?

2. Registre las razones que justifican la selección del contenido. ¿Por qué enseñar ese contenido?

3. Registre los objetivos de aprendizaje que considere pertinentes deben lograr los estudiantes asociados al contenido

4. Registre las razones que justifican la selección de los objetivos de aprendizaje.

5. Desarrolle una metodología para abordar el contenido en el aula, registre actividades, tareas, materiales, tiempos, los demás aspectos que considere necesarios.

6. Justifique las razones por las que considera pertinente la metodología propuesta.

7. Plantee criterios y estrategias de evaluación asociadas al contenido 